\title{
Formation of Au and tetrapyridyl porphyrin complexes in superfluid helium
}

Cheng Feng, Elspeth Latimer, Daniel Spence, Aula M. A. A. Al Hindawi, Shem Bullen, Adrian Boatwright, Andrew M. Ellis* and Shengfu Yang* Department of Chemistry, University of Leicester, Leicester LE1 7RH, UK

* Correspondence authors. Email addresses: andrew.ellis@,le.ac.uk; sfy1@le.ac.uk. Telephone +44 (0)116 252 2127; Fax +44 (0)116 2523789 .

Manuscript submitted to Phys. Chem. Chem. Phys. (Communications) 


\begin{abstract}
Binary clusters containing a large organic molecule and metal atoms have been formed by the co-addition of 5,10,15,20-Tetra(4-pyridyl)porphyrin (H2TPyP) molecules and gold atoms to superfluid helium nanodroplets, and the resulting complexes were then investigated by electron impact mass spectrometry. In addition to the parent ion H2TPyP yields fragments mainly from pyrrole, pyridine and methylpyridine ions because of the stability of their ring structures. When $\mathrm{Au}$ is co-added to the droplets the mass spectra are dominated by H2TPyP fragment ions with one or more Au atoms attached. We also show that by switching the order in which $\mathrm{Au}$ and H2TPyP are added to the helium droplets, different types of H2TPyP/Au complexes are clearly evident from the mass spectra. This study suggests a new route for the control over the growth of metal-organic compounds inside superfluid helium nanodroplets.
\end{abstract}




\section{Introduction}

Porphyrins are key components in many biological systems such as haemoglobin, chlorophyll and various cytochromes. As a result these molecules and their derivatives have long attracted the interest of biologists and biochemists. ${ }^{[1,2]}$ Porphyrins have good thermal stability and versatile chemical properties, making them candidates for a wide variety of applications such as catalysis, ${ }^{[3,4]}$ solar energy conversion, ${ }^{[5,6]}$ and the development of metal-organic frameworks. ${ }^{[7-9]}$ Among the porphyrins, 5,10,15,20-Tetra(4-pyridyl)porphyrin (H2TPyP) is one of the meso-substituted prophyrins that have been widely used in various chemical and photochemical studies. ${ }^{[10,11]}$ It has a strong Soret band at $\sim 420 \mathrm{~nm}$, depending on the precise composition of the molecule and metallocentric ad-atoms, ${ }^{[12]}$ which has enabled new applications such as dye-sensitized solar cells and thin semiconducting layers (e.g. organic LED displays). ${ }^{[13,14]}$ In addition, the pyridyl substituents can interact with a variety of reactive metal centres; H2TPyP can therefore be used as a building block for metal-organic architectures. ${ }^{[15-17]}$

In this report superfluid helium nanodroplets are employed as nanoreactors to explore the interaction between metal atoms and organic compounds. Helium nanodroplets are large helium clusters composed of typically $10^{3}-10^{8}$ helium atoms. ${ }^{[18,19]}$ These droplets have an equilibrium temperature of $0.37 \mathrm{~K}$ (for ${ }^{4} \mathrm{He}$ ) and they can pick up atoms/molecules with a near unit probability. ${ }^{[20]}$ When different types of atoms/molecules are added to the droplets, aggregation into binary complexes is expected. ${ }^{[21-30]}$ Here metal-organic complexes are formed by the co-addition of $\mathrm{Au}$ and H2TPyP to the droplets and any products are then deduced using mass spectrometry. Through these experiments we intend to show that different types of metal-organic compounds can be synthesized in helium nanodroplets by simply switching the order in which the dopants are added, i.e., the addition of Au to helium nanodroplets prior to or after the addition of H2TPyP.

\section{Experimental}

The apparatus employed is a UHV helium nanodroplet instrument which has been detailed elsewhere. ${ }^{[31-33]}$ Briefly, helium nanodroplets are formed by supersonic expansion of precooled helium gas through a $5 \mu \mathrm{m}$ pinhole nozzle and they then pass through a $0.5 \mathrm{~mm}$ skimmer to form a collimated helium nanodroplet beam. In the pickup region two oven evaporators, both of which are resistively heated, were used to add H2TPyP and Au separately to the droplets. The distance between the two oven evaporators is $\sim 10 \mathrm{~cm}$. The 
temperatures of the ovens are measured by C-type thermocouples. The pickup order can be changed by switching the oven positions. Further downstream a quadrupole mass spectrometer (Max-4000HT, Extrel), equipped with an electron ionization source operating at $70 \mathrm{eV}$, was used to measure mass spectra derived from the doped droplets.

In all of the experiments the helium source temperature was maintained at $12.5 \mathrm{~K}$ and the stagnation pressure was kept at 15 bar, producing droplets composed of $\sim 10^{4}$ helium atoms on average and with a velocity of $\sim 350 \mathrm{~m} / \mathrm{s}$. H2TPyP samples were purchased from Sigma-Aldrich with a stated purity of $>97 \%$ and were used without further purification. The oven temperature for H2TPyP was kept at $470 \mathrm{~K}$, which was optimized such that pickup of a single H2TPyP molecule was the most probable process. For Au the oven temperature was kept at $1260 \mathrm{~K}$. Under these conditions the largest $\mathrm{Au}$ cluster ions that can be seen in the mass spectra without the addition of H2TPyP contained more than $10 \mathrm{Au}$ atoms.

\section{Result and Discussion}

\section{H2TPyP mass spectrum}

To interpret the mass spectra of H2TPyP/Au complexes we first need to understand the mass spectrometry of H2TPyP in helium nanodroplets (see the inset of Figure 1 for the structure of H2TPyP). In previous work H2TPyP molecule has been added to helium droplets and then investigated via electronic spectroscopy; ${ }^{[34,35]}$ however no mass spectrum of H2TPYP embedded in superfluid helium droplets have been reported. The ionization process for molecules in helium nanodroplets is different from conventional electron ionization mass spectrometry of molecules in the gas phase. In helium droplets the most likely initial event is ionization of a helium atom. The resulting positive hole is mobile and can hop to adjacent helium atoms by a resonant charge transfer process. ${ }^{[36]}$ If the $\mathrm{He}^{+}$ion reaches an impurity after a small number of hops it can transfer its charge to the dopant. ${ }^{[37,38]}$ Otherwise selftrapping into helium cluster ions, dominated by $\mathrm{He}_{2}{ }^{+}$and leading to subsequent formation of an array of $\mathrm{He}_{n}{ }^{+}$ions, will result.

The mass spectrum from H2TPyP in helium droplets spanning the mass/charge range $50-1000$ is shown in Figure 1. Extensive fragmentation of the H2TPyP molecule is observed in the mass spectrum but the parent ion $(\mathrm{m} / \mathrm{z} 619)$ is clearly seen (marked as peak $\mathrm{H})$. The prominent fragments comprise clusters of peaks centred at $\mathrm{m} / \mathrm{z} 67,79,93,158,186$, 251 and 279. The clusters centred at $m / z$ 67, 79 and 93 (marked A, B and C) can be tentatively assigned to pyrrole $\left(\mathrm{C}_{4} \mathrm{NH}_{5}{ }^{+}\right)$, pyridine $\left(\mathrm{C}_{5} \mathrm{H}_{5} \mathrm{~N}^{+}\right)$, and methylpyridine $\left(\mathrm{C}_{6} \mathrm{H}_{7} \mathrm{~N}^{+}\right)$ 
ions. These are relatively stable ions on account of their ring structures. The larger clusters can then be assigned as combinations of these base units. For example, the $m / z 158$ channel corresponds to two pyridine units (D) and the $\mathrm{m} / \mathrm{z} 186$ species is consistent with two methylpyridine rings (E). The two heaviest ions, labelled as $F$ and $G$ in Figure 1, are consistent with a combination of three base fragments, i.e., two pyridines and one methylpyridine for the $m / z 251$ fragment (F), and three methylpyridines for $m / z 279$ (G).

Figure 2 shows expanded views of the spectra around the three base units. Here contributions from helium clusters ions, $\mathrm{He}_{n}{ }^{+}$, have been largely removed by subtracting a spectrum recorded without H2TPyP under otherwise identical conditions. As seen in the figure, these clusters also include $\mathrm{H}$-loss and/or proton addition channels, which are commonly observed in the mass spectra of organic molecules doped in superfluid helium nanodroplets, ${ }^{[39,40]}$ and in some cases the loss of two $\mathrm{H}$ atoms has been observed. One interpretation for efficient $\mathrm{H}$-loss is the low mass and small size of hydrogen atoms, which allows them to acquire high kinetic energy during charge transfer reactions between $\mathrm{He}^{+}$and H2TPyP and to escape from the droplets without recombination collisions. An alternative explanation is the presence of doubly charged reaction intermediates, which can contribute to the H-loss in organic molecules and biomolecules. ${ }^{[41]}$ The H-loss channel of the pyrrole ion at $\mathrm{m} / \mathrm{z} 66$ is as strong as the pyrrole ion signal at $\mathrm{m} / \mathrm{z} 67$. For the cluster centred at $\mathrm{m} / \mathrm{z} 79$, the protonated pyridine ion at $\mathrm{m} / \mathrm{z} 80$ is prominent and the H-loss product has nearly twice the abundance of the pyridine ion. For methylpyridine the protonated ion channel is the most prominent product, similar to pyridine. The high abundance of protonated pyridine and methylpyridine is likely due to the excess hydrogen atoms available when H2TPyP dissociates during the charge transfer ionization. In contrast, the protonated channel $(\mathrm{m} / \mathrm{z} 68)$ is absent for pyrrole, and the $\mathrm{H}_{2}$-loss channel of this cluster is significantly more intense than the corresponding ions in the pyridine and methylpyridine clusters. The formation of methylpyridine and pyrrole units both require cleavage of $\mathrm{C}-\mathrm{C}$ bonds between the methyl groups and neighbouring pyrrole units; hence the different fragmentation patterns suggest a competition between the pyrrole and the methylpyridine clusters in the ion fragmentation process. If we assume that ring fragments combine with $\mathrm{H}$ atoms through concerted reactions, the process must be in favour of transferring $\mathrm{H}$ atoms to the methylpyridine unit rather than pyrrole. For the fragments centred at $m / z 158,186,251$ and 279, the protonated channels are the most abundant products, which is unsurprising given that none of these ions contains a pyrrole unit. 
In the higher mass region of Figure 1 we see fragments of H2TPyP clusters attached to an intact H2TPyP molecule. The prominent fragments are centred at $m / z 712,776$ and 805 represent the combination of H2TPyP with the three base fragments identified for the H2TPyP monomer, as labelled in the figure. This suggests fragmentation into ring structures is also favoured in the ionization of $(\mathrm{H} 2 \mathrm{TPyP})_{N}$ clusters.

\section{H2TPyP/Au complexes}

Of particular interest in this work is to investigate how Au atoms are bonded to H2TPyP and how the structures of the H2TPyP/Au complexes are affected by the order in which the two dopants are added to the helium droplets. In previous experiments no elongated nanoparticles have been observed in helium droplets composed of $<10^{6}$ helium atoms, ${ }^{[31-33]}$ suggesting that quantized vortices, even if they exist, do not have significant influence on the clustering of H2TPyP and $\mathrm{Au}$ in this work. Therefore, the formation of Au-H2TPyP complexes is presumably driven by intermolecular forces. When $\mathrm{Au}$ is added to helium nanodroplets prior to H2TPyP the $\mathrm{Au}$ atoms will aggregate into $\mathrm{Au}_{n}$ clusters (on a timescale of a few $\mathrm{ns}^{[43]}$ ) before the helium nanodroplets reach the H2TPyP oven $(\sim 300 \mu \mathrm{s})$. Each H2TPyP/Au complex should therefore contain a small $\mathrm{Au}_{n}$ cluster and one or more H2TPyP molecules. $\mathrm{Au}$ atoms and small $\mathrm{Au}$ clusters can form dative bonds with the lone electron pair on a nitrogen atom; ${ }^{[44]}$ hence the Au clusters are likely to bond to one of the pyridyl nitrogen atoms, as seen in metal-H2TPyP supermolecular structures. ${ }^{[15-17]}$ In contrast, when the order of addition is reversed, different structures might result because all of the lone electron pairs on the four pyridyl nitrogen atoms are available for bonding. In addition, superfluid helium droplets are able to remove the binding energy released during the aggregation, allowing the structures to be stabilized with $\mathrm{Au}$ atoms bonded to different pyridyl nitrogen atoms.

To establish whether different structures for $\mathrm{H} 2 \mathrm{TPyP} / \mathrm{Au}$ complexes do result from a reversal of dopant pickup order we compare the H2TPyP/Au mass spectra obtained in the upper panels of Figure 3 in the range $m / z 190$ - 800. The upper panel of Figure 3 (a) was obtained with $\mathrm{Au}$ added prior to H2TPyP while for Figure 3 (b) the pickup order was reversed. Both spectra are dominated by Au cluster ions and binary cluster ions containing one $\mathrm{Au}$ atom and one or more fragments of H2TPyP. The fragment ions with gold attached are notably more abundant than the bare $\mathrm{Au}_{n}{ }^{+}$ions, especially in the upper panel of Figure 3(a). An obvious difference in the two spectra is seen for the Au-(methylpyridine) ${ }_{2}{ }^{+}$ion (the peaks marked as $\mathrm{Au}+\mathrm{E}$ in the upper panels of Figure 3), which is more prominent when $\mathrm{Au}$ was added to the droplets prior to H2TPyP. The formation of this ion involves two 
methylpyridine fragments, each requiring the cleavage of two $\mathrm{C}-\mathrm{C}$ bonds connecting to the neighbouring pyrrole units (see the structure of H2TPyP in the inset of Figure 1). This requires significant amount of energy being delivered to H2TPyP during the charge transfer process in order to allow the formation of this ion.

Here we tentatively interpret the observed difference in the mass spectra in term of energetics, which is closely related to the structures of H2TPyP/Au complexes. The binding energy between small Au clusters to the pyridyl nitrogen of H2TPyP is unknown. However, it is expected to be similar to the binding energy between $\mathrm{Au}_{n}$ clusters $(n=2-4)$ and pyridine $\left(\sim 1.1 \mathrm{eV}^{[44]}\right)$, which defines the energy that can be removed by the dissociation of a $\mathrm{Au}$ cluster from H2TPyP during the charge transfer ionization. The amount of energy that can be dissipated through this route depends on the number of sites for Au to bind with H2TPyP. As a result, if $\mathrm{Au}$ is bonded to H2TPyP at multiple sites the removal of multiple Au atoms from H2TPyP will help to quench the excess energy generated in the charge transfer reactions, leading to reduced fragmentation of H2TPyP.

Another effect of the bonding of $\mathrm{Au}$ atoms at multiple sites is a "fragmentation buffering" effect, which was proposed by Ren et al. in order to interpret the much reduced fragmentation of fragile organic molecules when co-embedded with polar molecules in helium droplets. ${ }^{[24,25]}$ It was found that when a polar molecule with large dipole moment was added to helium droplets, the charge-dipole interaction between $\mathrm{He}^{+}$and the polar molecule steers the $\mathrm{He}^{+}$towards the polar molecule. The consequence is that the fragmentation mainly occurs in the polar dopant rather than the organic molecule. For H2TPyP/Au complexes a similar interpretation can be applied. Although Au atoms and clusters do not possess dipole moments, they do have large polarizabilities. The mere fact of having Au at multiple sites will increase the probability of charge transfer from $\mathrm{He}^{+}$to the gold, rather than directly to the H2TPyP molecule. The porphyrin family generally has ionization energies lower than 7 $\mathrm{eV},{ }^{[45,46]}$ which is significantly lower than the ionization energy of atomic $\mathrm{Au}(\sim 9.2 \mathrm{eV})$. Therefore, if $\mathrm{Au}$ is first ionized by $\mathrm{He}^{+}$, secondary charge transfer between $\mathrm{Au}^{+}$and $\mathrm{H} 2 \mathrm{TPyP}$ might occur. This will deliver much less energy to H2TPyP as part of the excess energy can be removed by eliminating $\mathrm{Au}$ from $\mathrm{H} 2 \mathrm{TPyP}$ and/or the dissociation of small Au clusters. In effect the attachment of $\mathrm{Au}$ at multiple sites can introduce a quenching effect leading to the reduced fragmentation of $\mathrm{H} 2 \mathrm{TPyP}$.

In the lower panels of Figure 3 we present mass spectra of pure Au clusters in the $\mathrm{m} / \mathrm{z}$ range of $190-800$. Here $\mathrm{Au}_{2}{ }^{+}$is the most abundant product (the largest $\mathrm{Au}_{n}{ }^{+}$cluster ions 
contain more than $10 \mathrm{Au}$ atoms and are not shown in the figure). In a comparison with the spectra where H2TPyP has been added the mass spectra are clearly different. Regardless of the order in which the dopants are added even-numbered Au cluster ions are significantly reduced in abundance relative to odd-numbered ones when H2TPyP is added. This can be interpreted in term of the binding energy of Au clusters to H2TPyP. A single Au atom has one unpaired electron in the $6 s$ orbital and hence even-numbered Au clusters are closed-shell species which are likely to have a lower binding energy to H2TPyP when compared with the open-shell odd-numbered Au clusters. As a result, neutral even-numbered Au clusters will dissociate more easily from H2TPyP during the charge transfer process.

Finally, we investigate the influence of Au on the ionization of H2TPyP (see Figure 4 for the comparison between the spectra of H2TPyP and H2TPyP/Au complexes in the $m / z$ range of 60 - 200). Groups $\mathrm{A}$ and $\mathrm{B}$ correspond to the pyrrole and pyridine units, respectively, and yield structure in the mass spectrum that is nearly identical to that seen in the absence of $\mathrm{Au}$. For group $\mathrm{C}$, which derives from the methylpyridine channel, there is a significant increase in the $\mathrm{H}$-loss channel and a decrease in the protonated methylpyridine channel when $\mathrm{Au}$ is added. The decrease of protonated methylpyridine correlates with the prominent Au-attached fragments (see the upper panels in Figure 3 and later discussion), where $\mathrm{Au}$ replaces a hydrogen atom of the protonated methylpyridine. The same interpretation can also be applied to the group D fragments, which show a significant decline in the protonated channel. The influence of Au on the group E fragments, which derive from two methylpyridine units, is even more drastic. These cluster peaks disappear almost entirely from the H2TPyP/Au mass spectrum. As having been discussed above, this is explained by the attaching of $\mathrm{Au}$ atoms.

\section{Conclusions}

In this work we have formed binary clusters of $\mathrm{Au}$ and H2TPyP in superfluid helium nanodroplets for the first time. The mass spectrum of H2TPyP can be interpreted through structural stabilities of specific fragments and we have tentatively assigned the major fragments to simple heterocyclic units such as pyridine and pyrrole. The addition of Au to H2TPyP alters the relative abundances of fragments containing methylpyridine; in particular the Au reduces the signal for protonated methylpyridine, presumably by replacing the proton. For the pyridine and pyrrole groups Au has little influence. 
We have observed differences in the mass spectra of $\mathrm{H} 2 \mathrm{TPyP} / \mathrm{Au}$ complexes depending on the order in which $\mathrm{Au}$ and H2TPyP are added to the helium nanodroplets, suggesting that different types of H2TPyP/Au complexes have been formed. By adding Au to helium droplets prior to H2TPyP, a single Au cluster can bind to one of the lone pair of electrons of the pyridyl nitrogen of H2TPyP. When H2TPyP is added first, individual $\mathrm{Au}$ atoms can bind to any lone pair on the nitrogen atoms, allowing the formation of H2TPyP/Au complexes with multiple $\mathrm{Au}$ atoms at different bonding sites. If sufficient metal atoms are added, one can expect Au clusters to bind to all four nitrogen atoms and the size of the clusters can be controlled. In a recent experiment we have shown that chains of $\mathrm{Ag}$ nanoparticles formed in helium droplets can land on a substrate with no significant change on their spacing, which is clearly evident by subsequent addition of Si to the droplets after Ag. ${ }^{[33]}$ It therefore seems likely that metal cluster-porphyrin complexes with H2TPyP as the spacer between Au clusters will also be successfully soft-landed intact. This would further manipulation of the complexes and the construction of new materials. If the size of the metal clusters and the structures of the complexes can be well controlled, this could become a new route to form metal-cluster attached heterorganic compounds, although much more work is needed to confirm this. Potentially, the concept could be developed to construct metalorganic superstructures bridged by metal clusters rather than a single metal atom. ${ }^{[15-17]}$

\section{Acknowledgements}

SY and AME wish to thank the UK Engineering and Physical Sciences Research Council (EPSRC) and the Leverhulme Trust for grants in support of this work.

\section{References}

1. S. Mathai, T. A. Smith, K. P. Ghiggino, Photochem. Photobiol. Sci. 6, 995-1002 (2007).

2. H. L. Kee, J. Bhaumik, J. J. R. Diers, P. Mroz, M. R. Hamblin, D. F. Bocian, J. S. Lindsey, D. Holten, J. Photochem. Photobiol. A 200, 346-355 (2008).

3. C.-M. Che, J.-S. Huang, Chem. Commun. 3996-4015 (2009);

4. H. Lu, X. P. Zhang, Chem. Soc. Rev. 40, 1899-1909 (2011).

5. L. Li, E. W.-G. Diau, Chem. Soc. Rev. 42, 291-304 (2013). 
6. S. Mathew, A. Yella, P. Gao, R. Hamphry-Baker, B. E. F. Curchod, N. AshariAstani, I. Tavernelli, U. Rothlisberger, M. K. Nazeerudin, M. Grätzel, Nat. Chem. 6, 242-247 (2104).

7. M. H. Alkordi, Y. Liu, R. W. Larsen, J. F. Eubank, M. Eddaoudi, J. Am. Chem. Soc. 130, 12639-12641 (2008).

8. A. Fateeva, P. A. Chater, C. P. Ireland, A. A. Tahir, Y. Z. Khimyak, P. V. Wiper, J. R. Darwent, M. J. Rosseinsky, Angew. Chem. Int. Ed. 51, 7440-7444 (2012).

9. D. Deng, W. Chung, Z. Wei, Z. Gu, H. Jiang, Y. Chen, D. J. Darensbourg, H. Zhou, J. Am. Chem. Soc. 135, 17105-17110 (2013).

10. K. Kalyanasundaram, Inorg. Chem. 23, 2453-2459 (1984).

11. H. Kon, K. Tsuge, T. Imamura, Y. Sasaki, S. Ishizaka, N. Kitamura, Inorg. Chem. 45, 6875-6883 (2006).

12. N. M. Barbosa Neto, S. L. Oliveira, I. Guedes, L. R. Dinelli, L. Misoguti, C. R. Mendonça, A. A. Basista, S. C. Zílio, J. Braz. Chem. Soc. 17, 1377-1782 (2006).

13. A. Yella, H.-W. Lee, H. N. Tsao, C. Yi, A. K. Chandiran, M. Nazeeruddin, E. W.-G. Diau, C.-Y. Yeh, S. M. Zakeeruddin, M. Grätzel, Science 334, 629-634 (2011).

14. W. M. Campbell, K. W. Jolley, P. Wagner, K. Wagner, P. J. Walsh, K. C. Gordon, L. Schmidt-Mende, M. K. Nazeeruddin, Q. Wang, M. Grätzel, D. L. Officer, J. Phys. Chem. C 111, 11760-11762 (2007).

15. M. Kondo, Y. Kimura, K. Wada, T. Mizutani, Y. Ito, S. Kitagawa, Chem. Lett. 29, 818-819 (2000).

16. L. Carlucci, G. Ciani, D. M. Proserpio, F. Porta, Angew. Chem. Int. Ed. 42, 317-322 (2003).

17. I. Beletskaya, V. S. Tyurin, A. Y. Tsivadze, R. Guilard, C. Stern, Chem. Rev. 109, 1659-1713 (2009).

18. J. P. Toennies, A. F. Vilesov, Angew. Chem. Int. Ed. 43, 2622-2648 (2004).

19. R. E. Grisenti, J. P.Toennies, Phys. Rev. Lett. 90, 234501 (2003).

20. M. Lewerenz, B. Schilling, J. P. Toennies, J. Chem. Phys. 102, 8191-8207 (1995).

21. E. Lugovoj, J. P. Toennies, A. F. Vilesov, J. Chem. Phys. 112, 8217-8220 (2000).

22. M. Fárník, J. P. Toennies, J. Chem. Phys. 122, 014307 (2005).

23. J. M. Merritt, G. E. Douberly, P. L. Stiles, R. E. Miller, J. Phys. Chem. A 111,1230412316 (2007).

24. Y. Ren, R. Moro, V. V. Kresin, Eur. Phys. J. D 43, 109-112 (2007).

25. Y. Ren, V. V. Kresin, J. Chem. Phys. 128, 074303 (2008). 
26. S. Denifl, F. Zappa, I. Mähr, F. F. da Silva, A. Aleem, A. Mauracher, M. Probst, J. Urban, P. Mach, A. Bacher, O. Echt, T. D. Märk, P. Scheier, Angew. Chem. Intl. Ed. 48, 8940-8943 (2009).

27. H. Schöbel, C. Leidlmair, P. Bartl, A. Aleem, M. Hager, O. Echt, T. D. Märk, P. Scheier, Phys. Chem. Chem. Phys. 3, 1092-1098 (2011).

28. A. Gutberlet, G. Schwaab, O. Birer M. Masia, A. Kaczmarek, H. Forbert, M. Havenith, D. Marx, Science 324, 1545-1548 (2009).

29. J. Liu, B. Shepperson, A. M. Ellis, S. Yang, Phys. Chem. Chem. Phys. 13, 1392013925 (2011).

30. T. Liang, G. E. Douberly, Chem. Phys. Lett. 551, 54-59 (2012).

31. A. Boatwright, C. Feng, D. Spence, E. Latimer, C. Binns, A. M. Ellis, S. Yang, Faraday Disc. 162, 113-124 (2013).

32. E. Latimer, D. Spence, C. Feng, A. Boatwright, A. M. Ellis, S. Yang, Nano Lett. 14, 2902-2906 (2014).

33. D. Spence, E. Latimer, C. Feng, A. Boatwright, A. M. Ellis, S. Yang, Phys. Chem. Chem. Phys. 16, 6903-6906 (2014).

34. C. Callegari and W. Ernst, Handbook of High-resolution Spectroscopy, Ed. by M. Quack and F. Merkt, John Wiley, 2011; p 1551-1594.

35. R. Riechers, D. Pentlehner and A. Slenczka, Chem. Phys. 138, 244303 (2013).

36. K. R. Atkins, in Proceedings of the International School of Physics Enrico Fermi, Course XXI on Liquid Helium; G. Carerei, Ed.; Academic: New York, 1963; p 403.

37. A. M. Ellis and S. Yang, Phys. Rev. A 76, 032714 (2007).

38. W. K. Lewis, M. Lindsay, R. J. Bemish, R. E. Miller, J. Am. Chem. Soc. 127, 72357242 (2005).

39. S. Yang S. M. Brereton, M. D. Wheeler, A. M. Ellis J. Phys. Chem. A 110, 1791-1797 (2006).

40. S. Yang, S. M. Brereton, A. M. Ellis, Intl. J. Mass. Spectrosc. 253, 79-86 (2006).

41. S. Denifl, F. Zappa, I. Mähr, F. F. da Silva, A. Aleem, A. Mauracher, M. Probst, J. Urban, P. Mach, A. Bacher, O. Echt, T. D. Märk, P. Scheier, Angew. Chem. Int. Ed. 48, 8940-8943 (2009).

42. J. D. Mosley, A. M. Ricks, P. v. R. Schleyer, J. I. Wu, M. A. Duncan, J. Phys. Chem. A 116, 9689-9695 (2012).

43. A. W. Hauser, A. Volk, P. Thalera, W. E. Ernst, Phys. Chem. Chem. Phys. 17, 10805-10812 (2015) 
44. A. Martínez, J. Braz. Chem. Soc. 16, 337-344 (2005).

45. P. Dupuis, R. Roberge, C. Sandorfy, Chem. Phys. Lett. 75, 434-437 (1980).

46. O. Dolgounitcheva, V. G. Zakrzewski, J. V. Ortiz, J. Am. Chem. Soc. 127, 8240-8241 (2005). 
Figure Captions

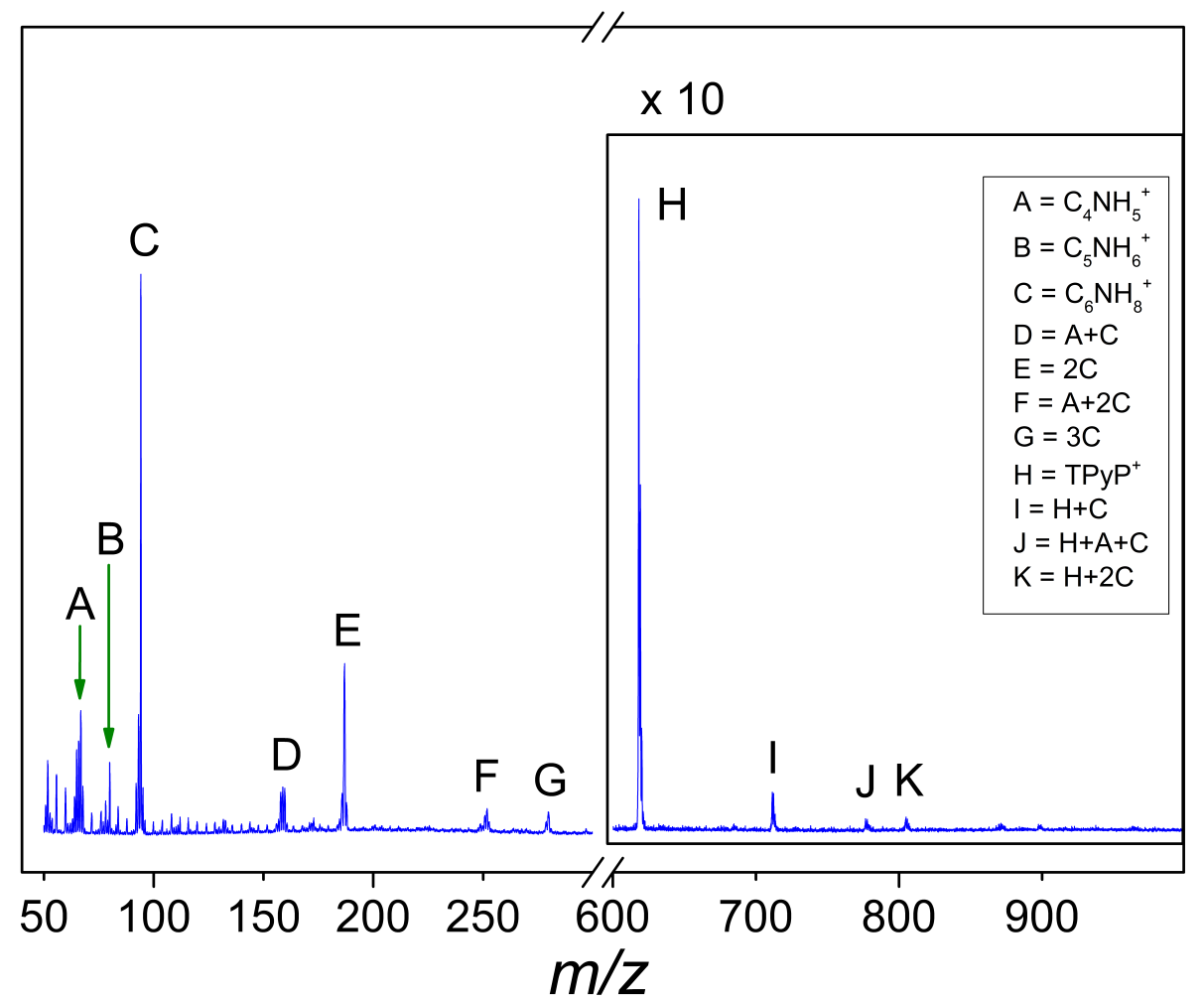

Figure 1 The mass spectrum of H2TPyP for $m / z 50-1000$. The inset shows the structure of H2TPyP molecule. 


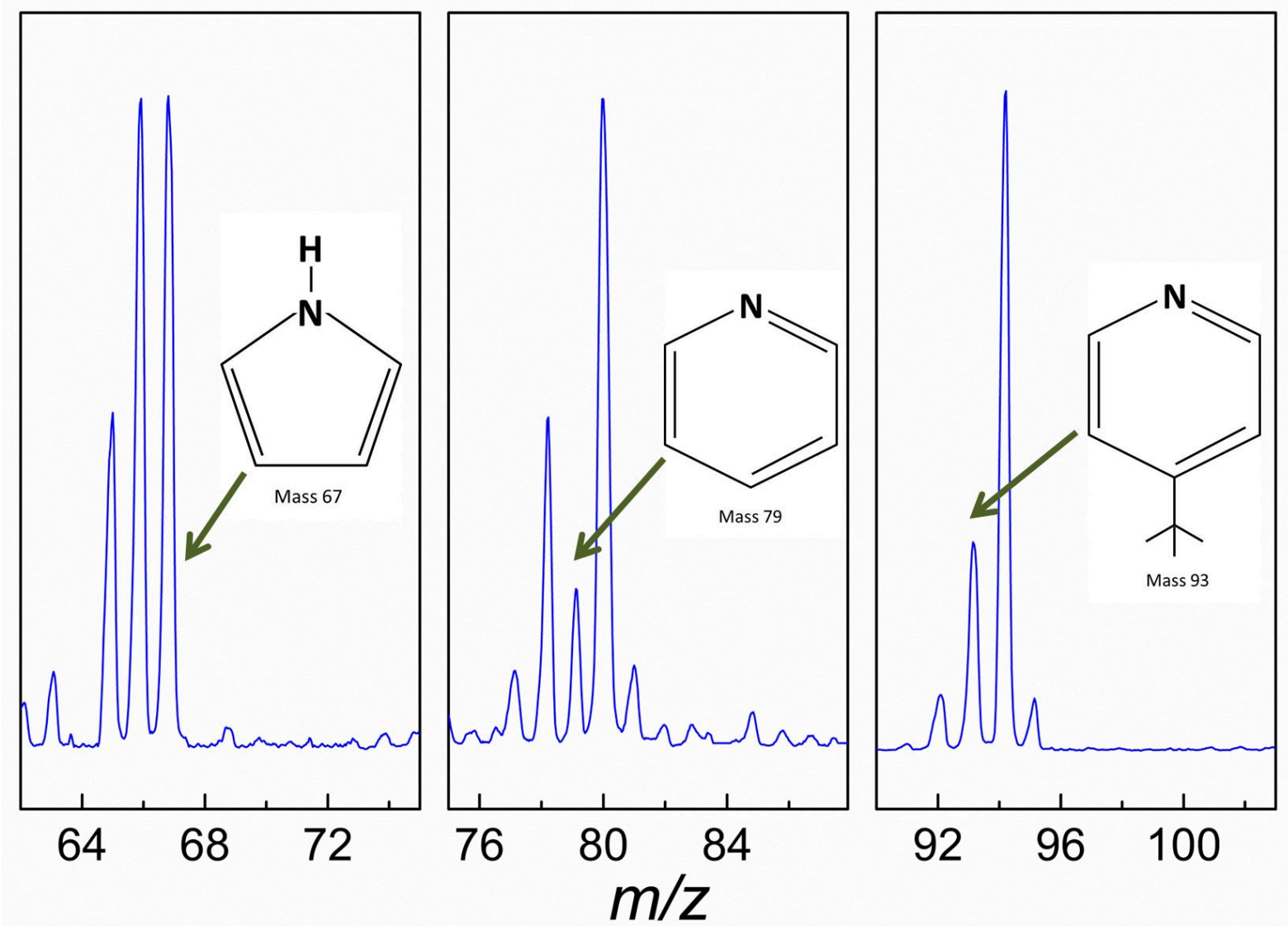

Figure 2 Expanded views of H2TPyP mass spectrum near the positions of three base fragments centred at a) $m / z$ 67(pyrrole); b) $m / z 79$ (pyridine) and c) $m / z 93$ (methylpyridine). 

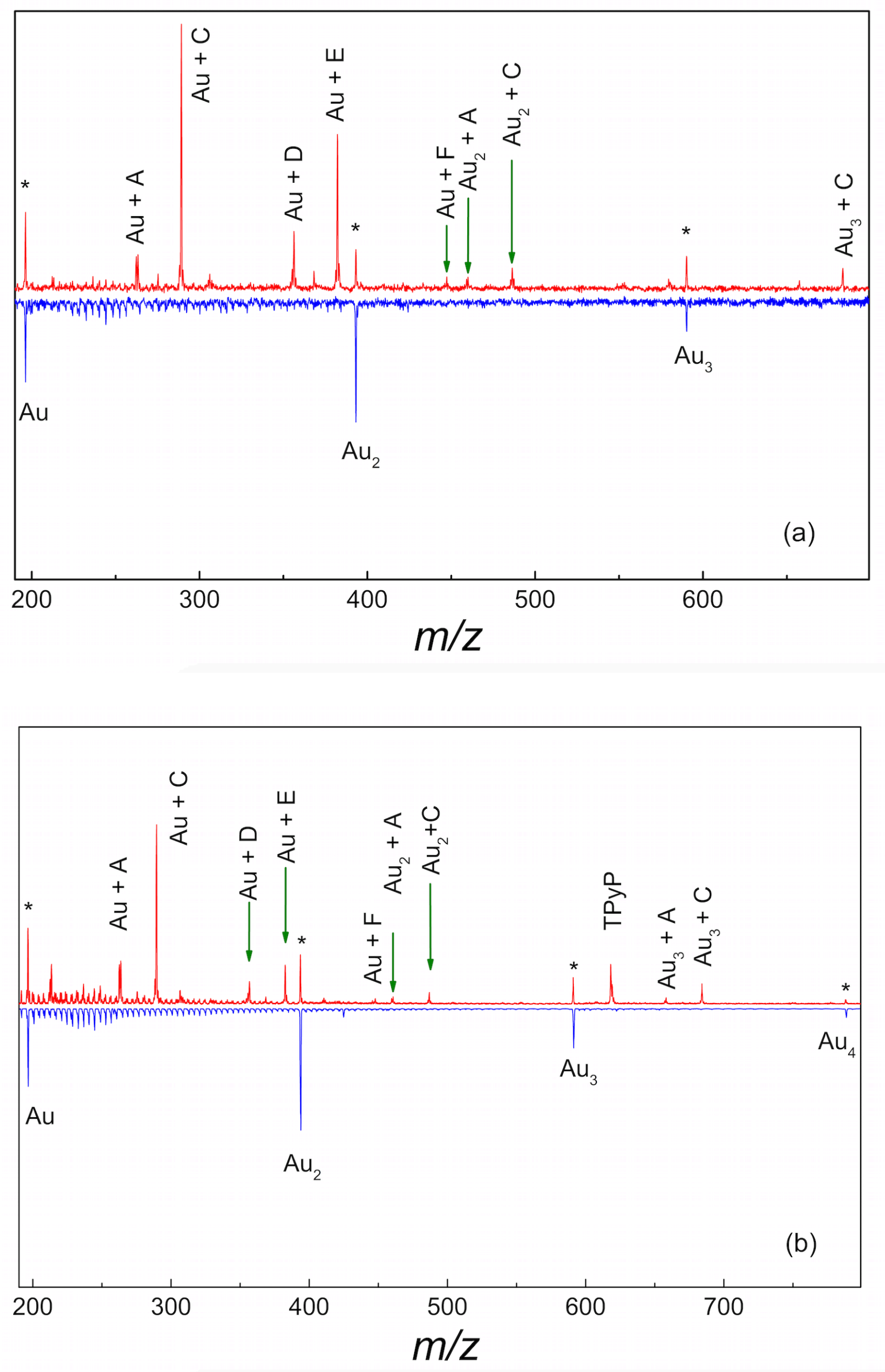
Figure 3 Comparison between the mass spectra of H2TPyP/Au complexes (upper panels) and Au clusters (lower panels). (a) Au added to helium droplets prior to H2TPyP; (b) Au added to helium droplets after H2TPyP. The mass spectra have been normalized to the $\mathrm{Au}^{+}$ion signal. The asterisks mark the $\mathrm{Au}_{n}{ }^{+}(n=1-4)$ ions.

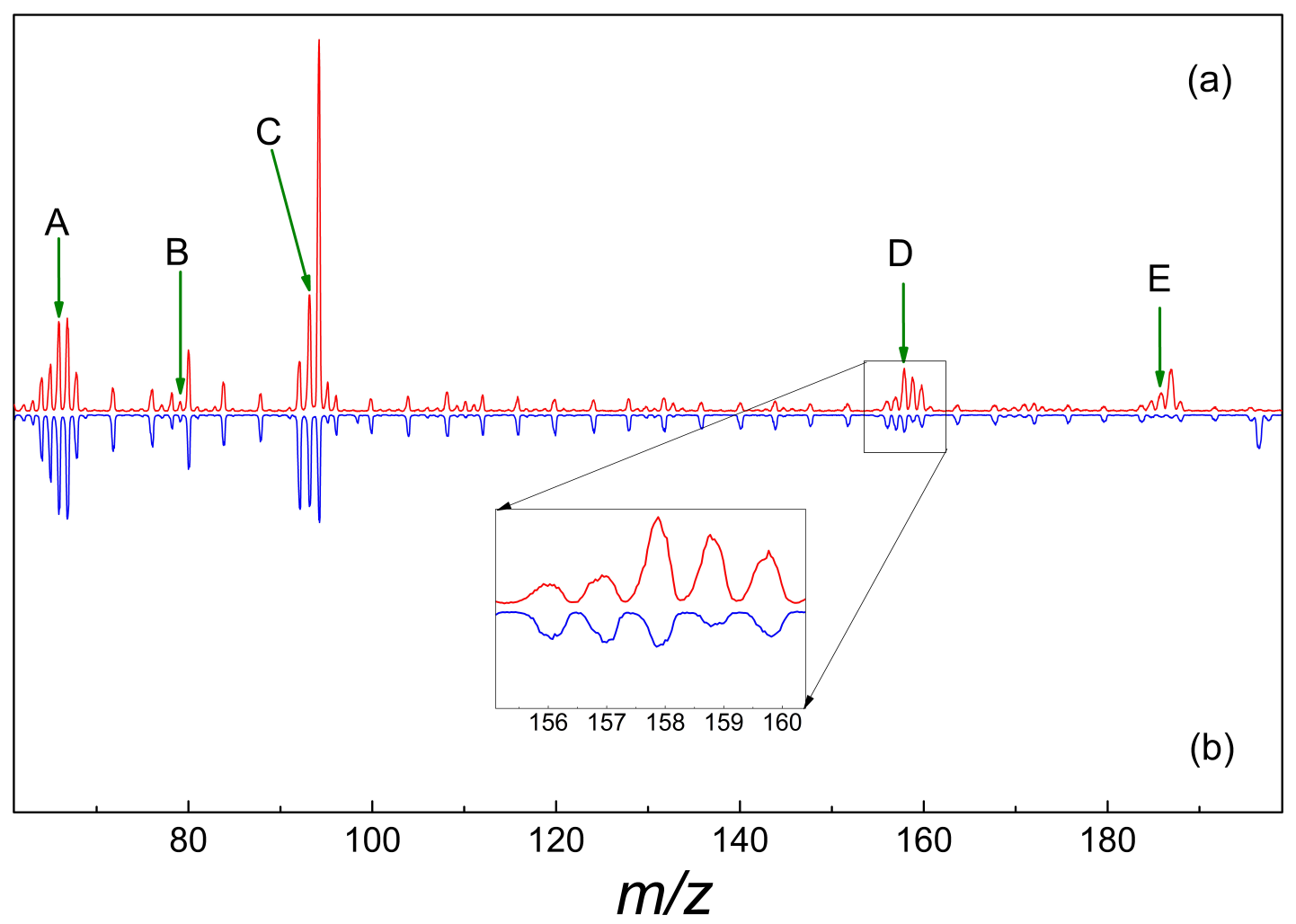

Figure 4 Influence of $\mathrm{Au}$ on the fragmentation pattern of H2TPyP. (a) The mass spectrum of H2TPyP; (b) The mass spectrum of H2TPyP/Au complexes. The inset shows the change of fragmentation pattern in the region of fragment $\mathrm{D}$, where the protonated fragment is negligible when Au is added. 